\title{
An Accelerated SOR-Like Method for Generalised Saddle Point Problems
}

\author{
Ting-Ting Feng ${ }^{1}$, Guo-Liang Chen ${ }^{1}$ and Xue-Ping Guo ${ }^{1, *}$ \\ ${ }^{1}$ Department of Mathematics, Shanghai Key Laboratory of Pure Mathematics and \\ Mathematical Practice, East China Normal University, Shanghai, China.
}

Received 26 October 2016; Accepted (in revised version) 26 August 2017.

\begin{abstract}
A recent accelerated SOR-like method for generalised saddle point problems is discussed. Sufficient conditions for convergence are derived, and some numerical experiments illustrate its effectiveness.
\end{abstract}

AMS subject classifications: 65F08, 65F10, 65F50

Key words: Accelerated SOR-like method, generalised saddle point problem, convergence.

\section{Introduction}

Generalised saddle point problems arise in constrained quadratic programming, constrained least squares problems, mixed finite-element approximations of elliptic PDEs, computational fluid dynamics, and Stokes problems $[1,6,9,10,22]$. Let $m, n$ be integers such that $m \geq n>0$. We consider the generalised saddle point problem

$$
\left(\begin{array}{cc}
A & B \\
B^{\top} & -C
\end{array}\right)\left(\begin{array}{l}
\mathbf{x} \\
\mathbf{y}
\end{array}\right)=\left(\begin{array}{l}
\mathbf{b} \\
\mathbf{q}
\end{array}\right)
$$

where $A \in \mathbb{R}^{m \times m}$ and $C \in \mathbb{R}^{n \times n}$ are respectively symmetric positive definite and symmetric positive semi-definite matrices, $B^{\top}$ is the transpose of a full column rank matrix $B \in \mathbb{R}^{m \times n}$, and $\mathbf{b} \in \mathbb{R}^{m}, \mathbf{q} \in \mathbb{R}^{n}$ are given vectors. In the special case $C=0$, the problem (1.1) obviously is reduced to the augmented system of linear equations

$$
\left(\begin{array}{cc}
A & B \\
B^{\boldsymbol{T}} & 0
\end{array}\right)\left(\begin{array}{l}
\mathbf{x} \\
\mathbf{y}
\end{array}\right)=\left(\begin{array}{l}
\mathbf{b} \\
\mathbf{q}
\end{array}\right)
$$

Various iteration methods have been used to solve such problems - e.g. Uzawa-type methods $[8,11,12,19,25]$, Hermitian and skew-Hermitian splitting (HSS) iteration [25], preconditioned Krylov subspace methods [1,21], restrictively preconditioned conjugate

${ }^{*}$ Corresponding author. Email addresses: tofengtingting@163.com (T.-T. Feng), glchen@math.ecnu . edu.cn (G.-L. Chen), xpguo@math.ecnu.edu.cn (X.-P. Guo) 
gradient methods $[6,23]$ and the successive overrelaxation (SOR) method [24], while Benzi et al. [10] reviewed existing approaches. For the augmented linear system (1.2), Golub et al. [16] proposed an SOR-like method, which was then further developed [7,13,14]. For the generalised saddle point problem (1.1), Cao [12] discussed the convergence of a nonlinear Uzawa algorithm; Bai \& Wang [8] studied parameterised inexact Uzawa methods (PIU), Zhou \& Zhang [25] proposed a generalisation of the parameterised inexact Uzawa methods (GPIU), and Huang \& Ma [18] developed a new GSOR method. Refs. [8, 18, 25] deal with symmetric positive definite matrices $C$, Miao \& Cao [19] discussed the GPIU method [25] for symmetric positive semidefinite matrices $C$ under the conditions $\operatorname{ker}(C) \cap \operatorname{ker}\left(B^{\boldsymbol{\top}}\right)=0$ with the rank $p$ of $C$ such that $0<p<n$.

Recently, Njeru \& Guo [20] have considered an accelerated SOR-like method (ASOR) for the augmented linear system (1.2). Here we apply the ASOR method to the generalised saddle point problem (1.1) when $C$ is a symmetric positive semidefinite matrix, consider properties of the eigenpairs of the iteration matrix, and establish sufficient convergence conditions for this method. In Section 2, we introduce the ASOR method for the problem (1.2), and discuss convergence for the generalised saddle point problem (1.1) in Section 3. Numerical experiments presented in Section 4 illustrate the efficiency of the method.

\section{The ASOR Method}

Let us rewrite the augmented linear system (1.2) as

$$
\left(\begin{array}{cc}
A & B \\
-B^{\top} & 0
\end{array}\right)\left(\begin{array}{l}
\mathbf{x} \\
\mathbf{y}
\end{array}\right)=\left(\begin{array}{c}
\mathbf{b} \\
-\mathbf{q}
\end{array}\right)
$$

with the coefficient matrix

$$
\mathscr{A}=\left(\begin{array}{cc}
A & B \\
-B^{\top} & 0
\end{array}\right)=D-L-U
$$

where

$$
D=\left(\begin{array}{cc}
\alpha A & 0 \\
0 & Q
\end{array}\right), \quad L=\left(\begin{array}{cc}
-A & 0 \\
B^{\top} & \frac{1}{2} Q
\end{array}\right), \quad U=\left(\begin{array}{cc}
\alpha A & -B \\
0 & \frac{1}{2} Q
\end{array}\right),
$$

involve a positive number $\alpha$ and a symmetric positive definite matrix $Q \in \mathbb{R}^{n \times n}$.

Let $\omega$ be a positive number. In the ASOR method we seek the solution of the problem (1.2) by the iteration scheme

$$
\begin{aligned}
& \mathbf{x}^{(k+1)}=\frac{\alpha}{\alpha+\omega} \mathbf{x}^{(k)}-\frac{\omega}{\alpha+\omega} A^{-1}\left(B \mathbf{y}^{(k)}-\mathbf{b}\right), \\
& \mathbf{y}^{(k+1)}=\mathbf{y}^{(k)}+\frac{2 \omega}{2-\omega} Q^{-1}\left(B^{\top} \mathbf{x}^{(k+1)}-\mathbf{q}\right),
\end{aligned}
$$


with the iteration matrix

$$
M_{\alpha, \omega}=\left(\begin{array}{cc}
\frac{\alpha}{\alpha+\omega} I_{m} & \frac{-\omega}{\alpha+\omega} A^{-1} B \\
\frac{2 \alpha \omega}{(\alpha+\omega)(2-\omega)} Q^{-1} B^{\top} & I_{n}-\frac{2 \omega^{2}}{(\alpha+\omega)(2-\omega)} Q^{-1} B^{\top} A^{-1} B
\end{array}\right) .
$$

As mentioned, this method was proposed by Njeru \& Guo [20], and was compared in the numerical experiments with SOR-like [16], GSOR [7] and GSSOR [13] methods. It was shown that the method converges if and only if $0<\omega<2$ and $\omega^{2} \mu_{\max } /(2-\omega)<2 \alpha+\omega$, where $\mu_{\max }$ is the largest eigenvalue of the matrix $Q^{-1} B^{\top} A^{-1} B$.

\section{The ASOR Method for the Generalised Saddle Point Problem and Its Convergence}

In this section, we study the ASOR method for the generalised saddle problem (1.1) and provide sufficient conditions for its convergence.

\subsection{The ASOR method for the problem (1.1)}

Analogously to Section 2, we rewrite the problem (1.2) as

$$
\mathscr{A} \mathbf{z}:=\left(\begin{array}{cc}
A & B \\
-B^{\top} & C
\end{array}\right)\left(\begin{array}{c}
\mathbf{x} \\
\mathbf{y}
\end{array}\right)=\left(\begin{array}{c}
\mathbf{b} \\
-\mathbf{q}
\end{array}\right)
$$

and represent the matrix $\mathscr{A}$ in the form $\mathscr{A}=D-L-U$, where

$$
D=\left(\begin{array}{cc}
\alpha A & 0 \\
0 & Q
\end{array}\right), \quad L=\left(\begin{array}{cc}
-A & 0 \\
B^{\top} & \frac{1}{2} Q
\end{array}\right), \quad U=\left(\begin{array}{cc}
\alpha A & -B \\
0 & \frac{1}{2} Q-C
\end{array}\right),
$$

with a positive number $\alpha$ and a symmetric positive definite matrix $Q \in \mathbb{R}^{n \times n}$.

Let $\omega$ be a positive number. The ASOR scheme for the generalised saddle problem (3.1) is

with the iteration matrix

$$
\left(\begin{array}{l}
\mathbf{x}^{(k+1)} \\
\mathbf{y}^{(k+1)}
\end{array}\right)=\mathscr{M}_{\alpha, \omega}\left(\begin{array}{l}
\mathbf{x}^{(k)} \\
\mathbf{y}^{(k)}
\end{array}\right)+\omega(D-\omega L)^{-1}\left(\begin{array}{c}
\mathbf{b} \\
-\mathbf{q}
\end{array}\right)
$$

$$
\begin{aligned}
\mathscr{M}_{\alpha, \omega} & =(D-\omega L)^{-1}((1-\omega) D+\omega U) \\
& =\left(\begin{array}{cc}
(\alpha+\omega) A & 0 \\
-\omega B^{\top} & \frac{2-\omega}{2} Q
\end{array}\right)^{-1}\left(\begin{array}{cc}
\alpha A & -\omega B \\
0 & \frac{2-\omega}{2} Q-\omega C
\end{array}\right) \\
& =\left(\begin{array}{cc}
\frac{1}{\alpha+\omega} A^{-1} & 0 \\
\frac{2 \omega}{(\alpha+\omega)(2-\omega)} Q^{-1} B^{\top} A^{-1} & \frac{2}{2-\omega} Q^{-1}
\end{array}\right)\left(\begin{array}{cc}
\alpha A & -\omega B \\
0 & \frac{2-\omega}{2} Q-\omega C
\end{array}\right) \\
& =\left(\begin{array}{cc}
\frac{\alpha}{\alpha+\omega} I_{m} \\
\frac{2 \alpha \omega}{(\alpha+\omega)(2-\omega)} Q^{-1} B^{\top} & I_{n}-\frac{2 \omega^{2}}{(\alpha+\omega)(2-\omega)} Q^{-1} B^{\top} A^{-1} B-\frac{2 \omega}{2-\omega} Q^{-1} C
\end{array}\right) .
\end{aligned}
$$


Consequently, the ASOR iteration scheme for the problem (1.1) is

$$
\begin{aligned}
& \mathbf{x}^{(k+1)}=\mathbf{x}^{(k)}+\frac{\omega}{\alpha+\omega} A^{-1}\left(\mathbf{b}-A \mathbf{x}^{(k)}-B \mathbf{y}^{(k)}\right), \\
& \mathbf{y}^{(k+1)}=\mathbf{y}^{(k)}+\frac{2 \omega}{2-\omega} Q^{-1}\left(B^{\boldsymbol{\top}} \mathbf{x}^{(k+1)}-C \mathbf{y}^{(k)}-\mathbf{q}\right) .
\end{aligned}
$$

Let us consider the matrix

$$
\mathscr{H}_{\alpha, \omega}=\frac{1}{\omega}(D-\omega L)=\left(\begin{array}{cc}
\frac{\alpha+\omega}{\omega} A & 0 \\
-B^{\top} & \frac{2-\omega}{2 \omega} Q
\end{array}\right) .
$$

Recalling symmetry and positive definiteness of matrices $A$ and $Q$, we note that $D-\omega L$ is invertible if and only if $\alpha+\omega \neq 0$ and $\omega \neq 2$. Now let us write

$$
\mathscr{A}=\mathscr{H}_{\alpha, \omega}-\mathscr{N}_{\alpha, \omega}
$$

where

$$
\mathscr{N}_{\alpha, \omega}=\left(\begin{array}{cc}
\frac{\alpha}{\omega} A & -B \\
0 & \frac{2-\omega}{2 \omega} Q-C
\end{array}\right) .
$$

It is readily seen that

$$
\mathscr{M}_{\alpha, \omega}=\mathscr{H}_{\alpha, \omega}^{-1} \mathscr{N}_{\alpha, \omega},
$$

so the ASOR method converges if and only if the spectral radius $\rho\left(\mathscr{M}_{\alpha, \omega}\right)$ of the matrix $\mathscr{M}_{\alpha, \omega}$ satisfies the inequality

$$
\rho\left(\mathscr{M}_{\alpha, \omega}\right)<1 \text {. }
$$

\subsection{Convergence of the ASOR method for the problem (1.1).}

Here we present sufficient conditions for the convergence of the ASOR method applied to the generalised saddle point problem (1.1). To do so, we consider the eigenpairs of the iteration matrix $\mathscr{M}_{\alpha, \omega}$.

Theorem 3.1. Assume that $A \in \mathbb{R}^{m \times m}, Q \in \mathbb{R}^{n \times n}$ are symmetric positive definite matrices, $B \in$ $\mathbb{R}^{m \times n}$ is a full column rank matrix, and $\mathbf{w}:=\left(\mathbf{u}^{*}, \mathbf{v}^{*}\right)^{*} \in \mathbb{C}^{m+n}$ is an eigenvector corresponding to the eigenvalue $\lambda$ of the iteration matrix $\mathscr{M}_{\alpha, \omega}$. Then $\lambda(\alpha+\omega)=\alpha$ or

$$
\lambda^{2}+\left[\frac{2 \omega^{2} \gamma}{(2-\omega)(\alpha+\omega)}-\frac{2 \alpha+\omega}{\alpha+\omega}+\frac{2 \omega}{2-\omega} \eta\right] \lambda+\left(1-\frac{2 \omega}{2-\omega} \eta\right)\left(\frac{\alpha}{\alpha+\omega}\right)=0,
$$

where

$$
\eta:=\eta(\mathbf{v})=\frac{\mathbf{v}^{*} C \mathbf{v}}{\mathbf{v}^{*} Q \mathbf{v}}, \quad \gamma:=\gamma(\mathbf{v})=\frac{\mathbf{v}^{*} B^{\top} A^{-1} B \mathbf{v}}{\mathbf{v}^{*} Q \mathbf{v}}
$$


Proof. The relation (3.4) implies

$$
\mathscr{N}_{\alpha, \omega} \mathbf{w}=\lambda \mathscr{H}_{\alpha, \omega} \mathbf{w}
$$

or

$$
\left(\begin{array}{cc}
\frac{\alpha}{\omega} A & -B \\
0 & \frac{2-\omega}{2 \omega} Q-C
\end{array}\right)\left(\begin{array}{l}
\mathbf{u} \\
\mathbf{v}
\end{array}\right)=\lambda\left(\begin{array}{cc}
\frac{\alpha+\omega}{\omega} A & 0 \\
-B^{\top} & \frac{2-\omega}{2 \omega} Q
\end{array}\right)\left(\begin{array}{l}
\mathbf{u} \\
\mathbf{v}
\end{array}\right)
$$

which leads to the system of equations

$$
\begin{aligned}
& (\alpha-\lambda \alpha-\lambda \omega) A \mathbf{u}=\omega B \mathbf{v}, \\
& ((1-\lambda)(2-\omega) Q-2 \omega C) \mathbf{v}=-2 \omega \lambda B^{\top} \mathbf{u} .
\end{aligned}
$$

We note that $\lambda \neq 1$ and $\mathbf{u} \neq 0$. Indeed, assuming $\lambda=1$ we obtain

$$
-A \mathbf{u}=B \mathbf{v}, \quad C \mathbf{v}=B^{\top} \mathbf{u},
$$

and simple transformations show that

$$
\left(C+B^{\top} A^{-1} B\right) \mathbf{v}=0 .
$$

Now $C+B^{\boldsymbol{\top}} A^{-1} B$ is a symmetric positive definite matrix, therefore the solution $\mathbf{v}$ of this homogeneous equation is trivial, and consequently the vector $\mathbf{u}=-A^{-1} B \mathbf{v}=0$ also. This contradicts the assumption that $\mathbf{w}$ is an eigenvector of $\mathscr{M}_{\alpha, \omega}$, hence $\lambda \neq 1$. The result $\mathbf{u} \neq 0$ can be proven similarly.

If $\alpha-\lambda \alpha-\lambda \omega=0$, then everything is proven. Assuming $\alpha-\lambda \alpha-\lambda \omega \neq 0$ and using the first equation in (3.7), we write $\mathbf{u}$ as

$$
\mathbf{u}=\frac{\omega}{\alpha-\lambda \alpha-\lambda \omega} A^{-1} B \mathbf{v}
$$

and substitute into the second equation to obtain

$$
((1-\lambda)(2-\omega) Q-2 \omega C) \mathbf{v}=-\frac{2 \omega^{2} \lambda}{\alpha-\lambda \alpha-\lambda \omega} B^{\top} A^{-1} B \mathbf{v} .
$$

It is easily seen that $\mathbf{v} \neq 0$ so that the term $\mathbf{v}^{*} Q \mathbf{v}$ is nonzero (since $Q$ is a positive definite matrix), hence on multiplying the above equation by $\mathbf{v}^{*} /\left(\mathbf{v}^{*} Q \mathbf{v}\right)$ from the left we obtain

$$
(\alpha-\lambda \alpha-\lambda \omega)[(\lambda-1)(2-\omega)+2 \omega \eta]=2 \omega^{2} \lambda \gamma,
$$

or

$$
\lambda^{2}+\left[\frac{2 \omega^{2} \gamma}{(2-\omega)(\alpha+\omega)}-\frac{2 \alpha+\omega}{\alpha+\omega}+\frac{2 \omega}{2-\omega} \eta\right] \lambda+\left(1-\frac{2 \omega}{2-\omega} \eta\right)\left(\frac{\alpha}{\alpha+\omega}\right)=0
$$

This completes the proof. 
Remark 3.1. If in addition to the assumptions of Theorem 3.1 the matrix $C$ is symmetric and positive semidefinite, then $\eta=\mathbf{v}^{*} C \mathbf{v} /\left(\mathbf{v}^{*} Q \mathbf{v}\right) \geq 0$. For $\eta=0$ and $\lambda(\alpha+\omega) \neq \alpha$, the eigenvalue $\lambda$ satisfies

$$
\lambda^{2}+\left[\frac{2 \omega^{2} \gamma}{(2-\omega)(\alpha+\omega)}-\frac{2 \alpha+\omega}{\alpha+\omega}\right] \lambda+\frac{\alpha}{\alpha+\omega}=0 .
$$

Remark 3.2. For the augmented system of linear equations (1.2), the parameter $\eta$ is equal to zero; and if $\lambda(\alpha+\omega) \neq \alpha$, then $\lambda$ satisfies the equation (3.8), consistent with Ref. [20].

Remark 3.3. In the special case $C=\delta Q, \delta>0$, one has $\eta=\delta$ and the representation (3.2) can be written as

$$
\left(\begin{array}{cc}
A & B \\
-B^{\top} & C
\end{array}\right)=\left(\begin{array}{cc}
\alpha A & 0 \\
0 & \frac{1}{\delta} C
\end{array}\right)-\left(\begin{array}{cc}
-A & 0 \\
B^{\top} & \frac{1}{2 \delta} C
\end{array}\right)-\left(\begin{array}{cc}
\alpha A & -B \\
0 & \left(\frac{1}{2 \delta}-1\right) C
\end{array}\right) .
$$

To consider the ASOR method for the problem (1.1), we need an auxiliary result.

Lemma 3.1 (cf. Young [24]). If $b$ and $c$ are real numbers, then the roots of the quadratic equation $x^{2}-b x+c=0$ are less than one in modulus if and only if $|c|<1$ and $|b|<1+c$.

We now proceed to derive convergence conditions for the ASOR method, on writing

$$
\eta_{\max }:=\max \eta(\mathbf{v}), \quad \gamma_{\max }:=\max \gamma(\mathbf{v}),
$$

where $\eta(\mathbf{v})$ and $\gamma(\mathbf{v})$ are defined in Eqs. (3.6).

Theorem 3.2. Let $A \in \mathbb{R}^{m \times m}, Q \in \mathbb{R}^{n \times n}$ be symmetric positive definite matrices and $B \in \mathbb{R}^{m \times n}$ be a full column rank matrix. If $\lambda(\alpha+\omega)=\alpha$, or if $\lambda(\alpha+\omega) \neq \alpha$ and the parameters $\eta, \alpha$ and $\omega$ satisfy one of the conditions

or

a) $\eta=0,0<\omega<2$ and $\frac{\omega^{2} \gamma_{\max }}{2-\omega}<2 \alpha+\omega$

b) $\eta>0,0<\omega<\frac{2}{\eta_{\max }+1}<2$, and also the inequality

$$
\left(\gamma_{\max }+\eta_{\max }+1\right) \omega^{2}+2\left(\alpha+\eta_{\max } \alpha-1\right) \omega-4 \alpha<0
$$

holds, then the iteration method (3.3) converges.

Proof. Since $\alpha>0$ and $\omega>0$, when $\lambda(\alpha+\omega)=\alpha$, the eigenvalue satisfies the inequality $|\lambda|=\alpha /(\alpha+\omega)<1$, so the iteration method converges.

For $\lambda(\alpha+\omega) \neq \alpha$ on the other hand, there are two cases to consider - viz. $\eta=0$ and $\eta>0$. If $\eta=0$, the related quadratic equation has the form (3.8), and the proof of the convergence is similar to that of Theorem 1 in Ref. [20]. Now we consider the other case 
$\eta>0$. In order to use Lemma 3.1 the coefficients of the equation (3.5) have to satisfy the inequalities

$$
\begin{aligned}
& \left|\left(1-\frac{2 \omega}{2-\omega} \eta\right)\left(\frac{\alpha}{\alpha+\omega}\right)\right|<1 \\
& \left|\frac{2 \omega^{2} \gamma}{(2-\omega)(\alpha+\omega)}-\frac{2 \alpha+\omega}{\alpha+\omega}+\frac{2 \omega}{2-\omega} \eta\right|<1+\left(1-\frac{2 \omega}{2-\omega} \eta\right)\left(\frac{\alpha}{\alpha+\omega}\right) .
\end{aligned}
$$

If

$$
\left|1-\frac{2 \omega}{2-\omega} \eta\right|<1
$$

then inequality (3.10) is fulfilled because $0<\alpha /(\alpha+\omega)<1$. This inequality (3.12) is equivalent to

$$
\left(\eta^{2}+\eta\right) \omega^{2}-2 \eta \omega<0 .
$$

Since $\omega>0, \eta>0$, the relation

$$
\omega<\frac{2}{1+\eta}<2,
$$

implies the inequality (3.10). The inequality (3.11) is equivalent to

$$
\begin{aligned}
-1-\left(1-\frac{2 \omega}{2-\omega} \eta\right)\left(\frac{\alpha}{\alpha+\omega}\right) & <\frac{2 \omega^{2} \gamma}{(2-\omega)(\alpha+\omega)}-\frac{2 \alpha+\omega}{\alpha+\omega}+\frac{2 \omega}{2-\omega} \eta \\
& <1+\left(1-\frac{2 \omega}{2-\omega} \eta\right)\left(\frac{\alpha}{\alpha+\omega}\right)
\end{aligned}
$$

so we rewrite the left-hand side as

$$
\frac{2 \omega^{2} \gamma+2 \omega^{2} \eta}{(2-\omega)(\alpha+\omega)}>0
$$

to validate the last inequality for all parameters $\omega \in(0,2)$ since $\gamma, \alpha$ and $\eta$ are positive. The right-hand side of inequality (3.15) implies

$$
\frac{2(\gamma+\eta+1) \omega^{2}+4(\alpha+\eta \alpha-1) \omega-8 \alpha}{(2-\omega)(\alpha+\omega)}<0,
$$

if

$$
(\gamma+\eta+1) \omega^{2}+2(\alpha+\eta \alpha-1) \omega-4 \alpha<0 .
$$

Consequently, from the condition (3.9) we conclude that the coefficients of the equation (3.5) satisfy all the conditions of Lemma 3.1 and the proof of Theorem 3.1 follows.

Remark 3.4. Theorem 3.2 shows that if $\eta>0, \omega\left(1+\eta_{\max }\right)<2$ and

$$
\alpha>\frac{2 \omega-\left(\gamma_{\max }+\eta_{\max }+1\right) \omega^{2}}{2 \omega+2 \eta_{\max } \omega-4},
$$

then the iteration method (3.3) converges. 


\section{Numerical Experiments}

The results of numerical experiments conducted on a PC equipped with $2.30 \mathrm{GHz} \mathrm{CPU}$ and 2GB RAM in the MATLAB 7.11.0 (R2010b) environment with the machine precision $\epsilon \approx 2.22 \times 10^{-16}$ are now discussed. In the tables below, IT means the number of iteration steps, CPU the CPU time (seconds), and RES the residual defined by

$$
\mathbf{R E S}:=\frac{\sqrt{\left\|\mathbf{b}-A \mathbf{x}^{(k)}-B \mathbf{y}^{(k)}\right\|_{2}^{2}+\| \mathbf{q}-B^{\top} \mathbf{x}^{(k)}+C \mathbf{y}^{(\mathbf{k}) \|_{2}^{2}}}}{\sqrt{\|\mathbf{b}\|_{2}^{2}+\|\mathbf{q}\|_{2}^{2}}},
$$

where $\left(\left(\mathbf{x}^{(k)}\right)^{\top},\left(\mathbf{y}^{(k)}\right)^{\top}\right)^{\boldsymbol{\top}}$ is the $k$-th iteration with the the starting point

$$
\left(\left(\mathbf{x}^{(0)}\right)^{\top},\left(\mathbf{y}^{(0)}\right)^{\top}\right)^{\top}=(0,0, \cdots, 0)^{\top} \in \mathbb{R}^{m+n} .
$$

Example 4.1. We consider the incompressible steady state Stokes problem in the square $D:=[-1,1] \times[-1,1][12]:$

$$
\left\{\begin{array}{l}
-v \Delta \mathbf{u}+\operatorname{grad} \mathbf{p}=f \\
\operatorname{div} \mathbf{u}=0
\end{array}\right.
$$

where $\Delta$ is the Laplace operator, $v$ denotes the viscosity of the fluid, $\mathbf{p}$ the pressure, and $\mathbf{u}$ the fluid velocity. The boundary conditions assumed are $\mathbf{u}_{x}=\mathbf{u}_{y}=0$ on the lines $x=-1$, $y=-1, x=1$ and $\mathbf{u}_{x}=1, \mathbf{u}_{y}=0$ on $y=1$.

The problem (4.1) was discretised using uniform square grids and the Incompressible Flow Iterative Solution Software (IFISS) developed by Elman et al. [15]. As finite elements, the bilinear constant velocity-pressure $Q_{1}-P_{0}$ pair with $\beta=0.25$ for local stabilisation was used. The resulting linear system has the form

$$
\left(\begin{array}{cc}
A & B \\
-B^{\top} & C
\end{array}\right)\left(\begin{array}{l}
\mathbf{x} \\
\mathbf{y}
\end{array}\right)=\left(\begin{array}{c}
\mathbf{b} \\
-\mathbf{q}
\end{array}\right)
$$

where $\mathbf{x}$ and $\mathbf{y}$ are the velocity and the pressure on the grid, respectively.

We compare the ASOR, SOR-like [16], and NSOR [18] methods for the problem (1.1). The iteration schemes are stopped when either RES $<10^{-9}$ or the iteration number 2500 is reached. For all methods, $Q$ is the approximate Schur complement matrix $B^{\top} P^{-1} B+C$ with $P=\operatorname{diag}(A)$. Table 1 contains the optimal parameters obtained in experiments. Table 1 and Fig. 1 show that for the problem (4.1) the ASOR method converges much faster than the two others. We note that the ASOR method is similar to the parameterized inexact Uzawa (PIU) method with the parameters $\omega^{\prime}=\alpha /(\alpha+\omega)$ and $\tau^{\prime}=2 \omega /(2-\omega)-$ cf. Ref. [8]. In our example, for both methods we used the same IT but slightly different CPUs, so the PIU results are not included in Table 1. Indeed, we recall that Hadjidimos [17] noted the equivalence of optimal relaxed block iterative methods for singular non-symmetric saddle point problems. 

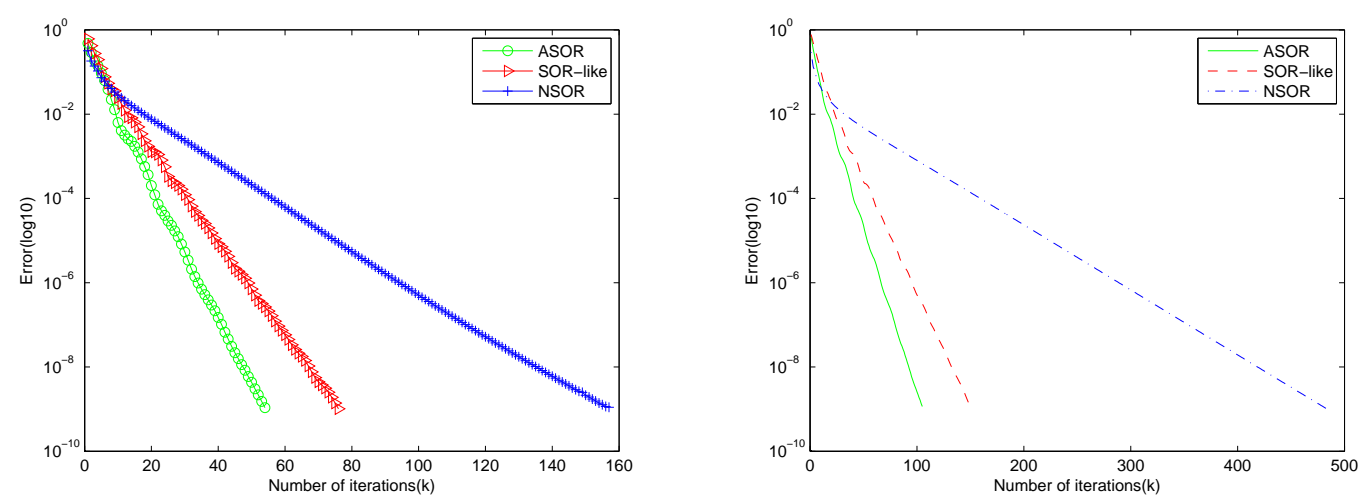

Figure 1: IT for the ASOR, SOR-like and NSOR methods with grids $8 \times 8$ and $16 \times 16$ in Example 4.1.

Table 1: IT, CPU and RES for the ASOR, SOR-like and NSOR methods in Example 4.1.

\begin{tabular}{|c|l|l|l|l|}
\hline Grid: & & $8 \times 8$ & $16 \times 16$ & $32 \times 32$ \\
\hline ASOR & $\omega_{\text {opt }}$ & 0.40 & 0.23 & 0.13 \\
& $\alpha_{\text {opt }}$ & 0.13 & 0.21 & 0.30 \\
& IT & 27 & 54 & 105 \\
& CPU & 0.0312 & 0.236 & 4.4303 \\
& RES & $5.421 \mathrm{e}-10$ & $7.616 \mathrm{e}-10$ & $9.413 \mathrm{e}-10$ \\
\hline SOR-like & $\omega_{\text {opt }}$ & 0.65 & 0.38 & 0.21 \\
& IT & 35 & 76 & 151 \\
& CPU & 0.0406 & 0.312 & 6.424 \\
& RES & $4.115 \mathrm{e}-10$ & $9.844 \mathrm{e}-10$ & $9.474 \mathrm{e}-10$ \\
\hline NSOR & $\omega_{\text {opt }}$ & 0.69 & 0.89 & 0.96 \\
& $\alpha_{\text {opt }}$ & 1.67 & 1.37 & 0.95 \\
& IT & 83 & 157 & 483 \\
& CPU & 0.0696 & 0.516 & 15.9308 \\
& RES & $6.891 \mathrm{e}-10$ & $9.932 \mathrm{e}-10$ & $9.947 \mathrm{e}-10$ \\
\hline
\end{tabular}

Example 4.2. We consider the generalised saddle point problem (1.1) with the following coefficient matrices $[18,25]$ :

$$
\begin{aligned}
& A=\left(\begin{array}{cc}
I \otimes T+T \otimes I & 0 \\
0 & I \otimes T+T \otimes I
\end{array}\right) \in \mathbb{R}^{2 p^{2} \times 2 p^{2}}, \\
& B=\left(\begin{array}{c}
I \otimes F \\
F \otimes I
\end{array}\right) \in \mathbb{R}^{2 p^{2} \times p^{2}}, \quad C=I \in \mathbb{R}^{p^{2} \times p^{2}}, \\
& T=\frac{1}{h} \cdot \operatorname{tridiag}(-1,2,-1) \in \mathbb{R}^{p \times p}, \quad F=\frac{1}{h} \cdot \operatorname{tridiag}(-1,1,0) \in \mathbb{R}^{p \times p},
\end{aligned}
$$

where $h=1 /(p+1)$ denotes the mesh size, $\otimes$ the Kronecker product, and $U=\operatorname{tridiag}(a, b, c)$ is the tridiagonal matrix with nonzero entries $u_{i, i-1}=a, u_{i, i}=b, u_{i, i+1}=c$.

We set $m=2 p^{2}, n=p^{2}$, so the total number of unknowns is $m+n=3 p^{2}$. Moreover, the right-hand-side $\left(\mathbf{b}^{\top}, \mathbf{q}^{\top}\right)^{\top} \in \mathbb{R}^{m+n}$ is the image of the vector $\left(\left(\mathbf{x}_{*}\right)^{\top},\left(\mathbf{y}_{*}\right)^{\top}\right)^{\top}=(1,1, \cdots, 1)^{\top} \in$ 

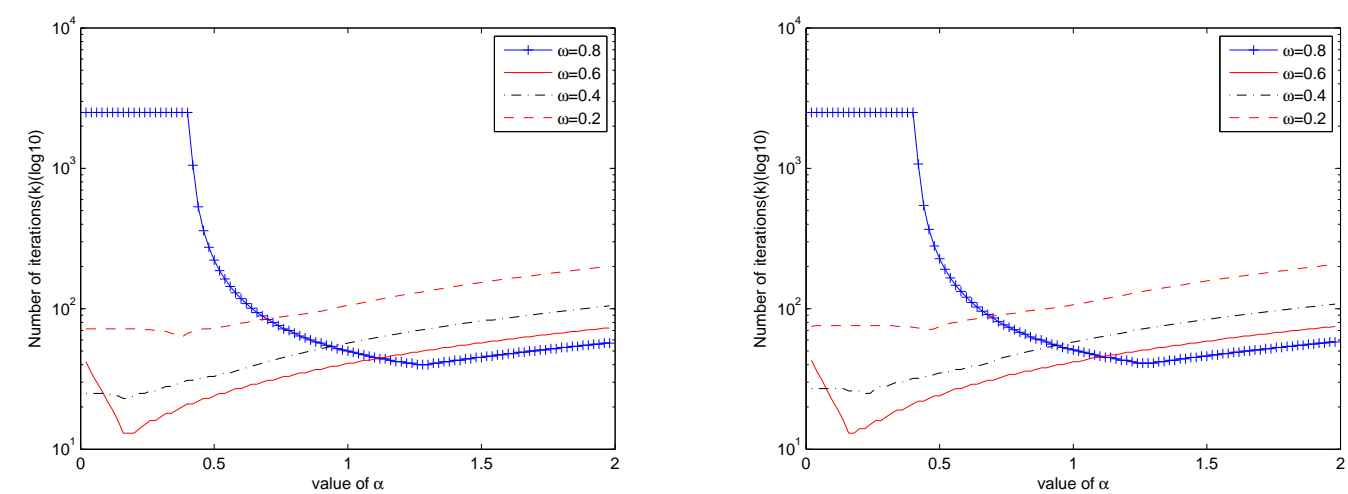

Figure 2: IT for the ASOR method with respect to $\alpha$ and $\omega$ for $p=16$ and $p=32$ in Example 4.2.

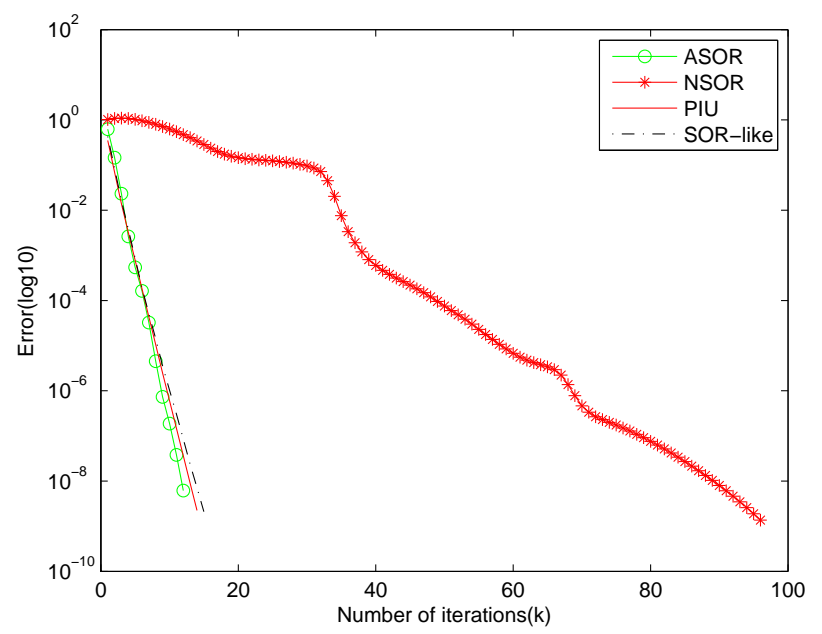

Figure 3: Number of iterations for ASOR, PIU, SOR-like and NSOR methods $(p=16)$ in Example 4.2.

$\mathbb{R}^{m+n}$ under the mapping (1.1). The iteration is stopped when either

$$
E R R=\frac{\sqrt{\left\|\mathbf{x}^{(k)}-\mathbf{x}_{*}\right\|_{2}^{2}+\left\|\mathbf{y}^{(k)}-\mathbf{y}_{*}\right\|_{2}^{2}}}{\sqrt{\left\|\mathbf{x}^{(0)}-\mathbf{x}_{*}\right\|_{2}^{2}+\left\|\mathbf{y}^{(0)}-\mathbf{y}_{*}\right\|_{2}^{2}}}<10^{-9}
$$

or the iteration number 2500 is reached.

We compare the ASOR, PIU [8], SOR-like [16], and NSOR [18] methods for the problem (1.1). In this example, we set $Q=C$ for (3.3), similarly to NSOR method in Ref. [18], but in the PIU and SOR-like methods we follow the settings in Ref. [8] and put $P=A$ and $Q=B^{\boldsymbol{\top}} P^{-1} B+C$. For all methods, the experimental optimal parameters $\alpha$ and $\omega$ are used. The results presented in Table 2 and Figs. 2 and 3 show the efficiency of our method. 
Table 2: IT, CPU and RES for the ASOR, PIU, SOR-like and NSOR methods in Example 4.2.

\begin{tabular}{|c|l|l|l|l|l|l|}
\hline $\mathrm{p}$ & & 16 & 24 & 32 & 40 & 48 \\
\hline $\mathrm{m}$ & & 512 & 1052 & 2048 & 3200 & 4608 \\
$\mathrm{n}$ & & 256 & 576 & 1024 & 1600 & 2304 \\
$m+n$ & & 768 & 1728 & 3072 & 4800 & 6912 \\
\hline ASOR & $\omega_{\text {opt }}$ & 0.58 & 0.58 & 0.58 & 0.58 & 0.58 \\
& $\alpha_{\text {opt }}$ & 0.14 & 0.14 & 0.14 & 0.14 & 0.14 \\
& $\mathrm{IT}$ & 12 & 12 & 12 & 13 & 13 \\
& $\mathrm{CPU}$ & 0.0328 & 0.158 & 0.495 & 1.278 & 2.735 \\
& RES & $4.905 \mathrm{e}-10$ & $5.137 \mathrm{e}-10$ & $4.833 \mathrm{e}-10$ & $1.161 \mathrm{e}-10$ & $1.135 \mathrm{e}-10$ \\
\hline PIU & $\omega_{\text {opt }}$ & 0.88 & 0.86 & 0.86 & 0.58 & 0.86 \\
& $\alpha_{\text {opt }}$ & 0.83 & 0.83 & 0.83 & 0.83 & 0.84 \\
& IT & 14 & 15 & 16 & 16 & 16 \\
& CPU & 0.0515 & 0.256 & 0.817 & 2.047 & 4.313 \\
& RES & $5.262 \mathrm{e}-10$ & $3.718 \mathrm{e}-10$ & $1.081 \mathrm{e}-10$ & $1.180 \mathrm{e}-10$ & $2.688 \mathrm{e}-10$ \\
\hline SOR-like & $\omega_{\text {opt }}$ & 0.85 & 0.84 & 0.84 & 0.84 & 0.84 \\
& IT & 15 & 16 & 16 & 16 & 16 \\
& CPU & 0.0452 & 0.267 & 0.819 & 2.050 & 4.322 \\
& RES & $6.199 \mathrm{e}-10$ & $1.727 \mathrm{e}-10$ & $2.915 \mathrm{e}-10$ & $2.782 \mathrm{e}-10$ & $2.688 \mathrm{e}-10$ \\
\hline NSOR & $\omega_{\text {opt }}$ & 1.58 & 1.64 & 1.72 & 1.78 & 1.80 \\
& $\alpha_{\text {opt }}$ & 0.24 & 0.19 & 0.16 & 0.13 & 0.10 \\
& IT & 96 & 150 & 178 & 222 & 275 \\
& CPU & 0.0515 & 0.429 & 1.420 & 4.065 & 9.850 \\
& RES & $4.307 \mathrm{e}-10$ & $3.244 \mathrm{e}-10$ & $1.254 \mathrm{e}-10$ & $2.487 \mathrm{e}-10$ & $1.668 \mathrm{e}-10$ \\
\hline
\end{tabular}

\section{Conclusion}

We have applied the ASOR method [20] to the generalised saddle point problem (1.1) with a symmetric positive semidefinite matrix, and provided sufficient conditions for its convergence.

\section{Acknowledgments}

We express our sincere thanks to the reviewers and editor for insightful suggestions that improved the presentation in this article significantly. We also thank Dr. Victor Didenko for carefully reading and amending a previous version of this article. One of us (G.-L. C.) is supported by a National Natural Science Foundation of China Grant 11471122, and the other (X.-P. G.) is supported in part by National Natural Science Foundation of China Grants 11371145 and 11471122, and by the Science and Technology Commission of Shanghai Municipality (Grant 13dz2260400).

\section{References}

[1] Z.-Z. Bai, Structured preconditioners for nonsingular matrices of block two-by-two structures, Math. Comp. 75, 791-815 (2006). 
[2] Z.-Z. Bai and G. H. Golub, Accelerated Hermitian and skew-Hermitian splitting iteration methods for saddle-point problems, IMA J. Numer. Anal. 27, 1-23 (2007).

[3] Z.-Z. Bai, G. H. Golub and C.-K. Li, Optimal parameter in Hermitian and skew-Hermitian splitting method for certain two-by-two block matrices, SIAM J. Sci. Comput. 28, 583-603 (2006).

[4] Z.-Z. Bai, G. H. Golub and M. K. Ng, Hermitian and skew-Hermitian splitting methods for nonHermitian positive definite linear systems, SIAM J. Matrix. Anal. Appl. 24, 603-626 (2003).

[5] Z.-Z. Bai, G. H. Golub and J.-Y. Pan, Preconditioned Hermitian and skew-Hermitian splitting methods for non-Hermitian positive semidefinite linear system, Numer. Math. 98, 1-32 (2004).

[6] Z.-Z. Bai and G.-Q. Li, Restrictively preconditioned conjugate gradient methods for systems of linear equations, IMA J. Numer. Anal. 23, 561-580 (2003).

[7] Z.-Z. Bai, B. N. Parlett and Z.-Q. Wang, On generalized successive overrelaxation methods for augmented linear systems, Numer. Math. 102, 1-38 (2005).

[8] Z.-Z. Bai and Z.-Q. Wang, On parameterized inexact Uzawa methods for generalized saddle point problems, Linear Algebra Appl. 428, 2900-2932 (2008).

[9] M. Benzi and G. H. Golub, A preconditioner for generalized saddle point problems, SIAM J. Matrix Anal. Appl. 26, 20-41 (2004).

[10] M. Benzi, G. H. Golub and J. Liesen, Numerical solution of saddle point problems, Acta Numer. 14, 1-137 (2005).

[11] J. H. Bramble, J. E. Pascisk and A. T. Vassilev, Analysis of the inexact Uzawa algorithm for saddle point problems, SIAM J. Numer. Anal. 34, 1072-1092 (1997).

[12] Z.-H. Cao, Fast Uzawa algorithm for generalized saddle point problems, Appl. Numer. Math. 46, 157-171 (2003).

[13] Z. Chao, N. Zhang and Y. Lu, Optimal parameters of the generalized symmetric SOR method for augmented systems, J. Comput. Appl. Math. 266, 52-60 (2014).

[14] M. T. Darvishi and P. Hessari, Symmetric SOR method for augmented systems, Appl. Math. Comput. 183, 409-415 (2006).

[15] H. C. Elman, A. Ramage and D. J. Silvester, Algorithm 866: IFISS, a Matlab toolbox for modelling incompressible flow, ACM Trans. Math. Software 33, 1-18 (2007).

[16] G. H. Golub, X. Wu and J.-Y. Yuan, SOR-like method for augmented systems, BIT Numer. Math. 41, 71-85 (2001).

[17] A. Hadjidimos, On equivalence of optimal relaxed block iterative methods for the singular nonsymmetric saddle point problem, Linear Algebra Appl. 522, 175-202 (2017).

[18] N. Huang and C.-F. Ma, A new GSOR method for generalised saddle point problems, East Asian J. Appl. Math. 6, 23-41 (2016).

[19] S.-X. Miao and Y. Cao, A note on GPIU method for generalized saddle point problems, Appl. Math. Comput. 230, 27-34 (2014).

[20] P. N. Njeru and X.-P. Guo, Accelerated SOR-like method for augmented linear systems, BIT Numer. Math. 56, 557-571 (2016).

[21] J.-Y. Pan, M. K. Ng and Z.-Z. Bai, New preconditioners for saddle point problems, Appl. Math. Comput. 172, 762-771 (2006).

[22] I. Perugia and V. Simoncini, Block-diagonal and indefinite symmetric preconditioners for mixed finite element formulations, Numer. Linear Algebra Appl. 7, 585-616 (2000).

[23] J.-F. Yin and Z.-Z. Bai, The restrictively preconditioned conjugate gradient methods on normal residual for two-by-two linear systems, J. Comput. Math. 26, 240-249 (2008).

[24] D. M. Young, Iterative Solutions of Large Linear Systems, Academic Press (1971).

[25] G.-F. Zhang and S.-S. Wang, A generalization of parameterized inexact Uzawa method for generalized saddle point problems, Appl. Math. Comput. 215, 599-607 (2009). 